\title{
A CONSTRUCTION OF PROJECTIVE BASES FOR IRREDUCIBLE REPRESENTATIONS OF MULTIPLICATIVE GROUPS OF DIVISION ALGEBRAS OVER LOCAL FIELDS
}

\author{
David Kazhdan
}

\begin{abstract}
Let $F_{0}$ be a local non-archimedian field of positive characteristic, $D_{0}$ be a skew-field with center $F_{0}$ and $G_{0}:=D_{0}^{\star}$ be the multiplicative group of $D_{0}$. The goal of this paper is to provide a canonical decomposition of any complex irreducible representation $V$ of $G_{0}$ in a direct sum of one-dimensional subspaces.
\end{abstract}

Let $k=\mathbb{F}_{q}$ be a finite field, $F:=k(t)$ the field of rational functions on the projective line $\mathbb{P}^{1}$ over $k$. Let $S$ be the set of points of $\mathbb{P}^{1}$. For any point $s \in S$ we denote by $F_{s}$ the completion of $F$ at $s$, by $\nu_{s}: F_{s} \rightarrow \mathbb{Z} \cup+\infty$ the valuation map and by $\mathcal{O}_{s} \subset F_{s}$ the subring of integers. We denote by $\mathbb{A}$ the ring of adeles for $F$.

Let $D$ be a skew-field with center $F$ unramified outside $\{0, \infty\}, D_{0}:=$ $D \otimes_{F} F_{0}$ and $D_{\infty}:=D \otimes_{F} F_{\infty}$. We have $\operatorname{dim}_{F}(D)=n^{2}$.

We denote by $\underline{G}$ the mutiplicative group of $D$ considered as an algebraic $F$-group, write $G_{s}:=\underline{G}\left(F_{s}\right)$ and denote by $\left.\mathbb{C}_{c} G_{0}\right) \subset \mathbb{C}\left(G_{0}\right)$ the algebra of locally constant compactly supported functions on $G_{0}$.

For any point $s \in S, s \neq 0, \infty$ we identify the group the group $\underline{G}\left(F_{s}\right)$ with $G l_{n}\left(F_{s}\right)$ and define $K_{s}:=G l_{n}\left(\mathcal{O}_{s}\right)$.

Let $N_{\infty}: G_{\infty} \rightarrow F_{\infty}^{\star}$ be the reduced norm. We define $K \infty=\{g \in$ $\left.D_{\infty} \mid \nu_{\infty}\left(N_{\infty}(g)\right) \geq 0\right\}$ and $K_{\infty}^{1}=\left\{g \in D_{\infty} \mid \nu_{\infty}\left(N_{\infty}(g-1)\right)>0\right\}$. Then $K_{\infty} \subset G_{\infty}$ is an open compact subgroup and $K_{\infty} / K_{\infty}^{1}=\mathbb{F}_{q^{n}}^{\star}$. We define $K^{1}:=\prod_{s \in S-\{0, \infty\}} K_{s} \times K_{\infty}^{1}$.

The multiplication defines a map

$$
\kappa: G_{0} \times K^{1} \times \underline{G}(F) \rightarrow \underline{G}(\mathbb{A})
$$

This paper is based on the following result

Proposition 0.1. The map $\kappa$ is a bijection.

Proof. The surjectivity follows from Lemma 7.4 in [2]. To show the injectivity it is sufficient to check the equality

$$
\left(D_{0}^{*} \times K^{1}\right) \cap G_{E}=\{e\}
$$

which is obvious. 
Let $R$ be the space of $\mathbb{C}$-valued locally constant functions on $\underline{G}(\mathbb{A}) / \underline{G}(F) K^{1}$, $\mathcal{H}_{s}, s \neq 0, \infty$ be the spheical Hecke algebra at $s, \mathcal{H}:=\prod_{s \in S-\{0, \infty\}} \mathcal{H}_{s}$.

We have a natural action $a \rightarrow \hat{a}$ of the commutative algebra $\mathcal{A}:=$ $\mathcal{H} \otimes \mathbb{C}\left[K_{\infty}^{0} / K_{\infty}^{1}\right]$ on $R$.

Corollary 0.2. (1) The natural action of the group $G_{0}$ on the space $X:=\underline{G}(\mathbb{A}) / K^{1} \times \underline{G}(F)$ is simply transitive. So we can identify $X$ with $G_{0}$.

(2) The restriction to $G_{0}$ defines a $G_{0}$-equivariant isomorphism $u$ : $R \rightarrow \mathbb{C}\left(G_{0}\right)$.

(3) For any irreducible representation $V$ of $G_{0}$ the restriction to $G_{0}$ defines an isomorphism $u_{V}: \operatorname{Hom}_{G_{0}}\left(V^{\vee}, L\right) \rightarrow V$ where $V^{\vee}$ is the representation dual to $V$.

(4) There exists a map $\alpha: \mathcal{A} \rightarrow \mathbb{C}_{c}\left(G_{0}\right)$ such that

$$
f \star \alpha(a))=\hat{a}(f), a \in \mathcal{A}, f \in \mathbb{C}_{c}\left(G_{0}\right)
$$

Let $\Xi$ be the set of homomorphisms $\chi: \mathcal{H} \otimes \mathbb{C}\left[\mathbb{F}_{q^{n}}^{\star}\right] \rightarrow \mathbb{C}$. For any $\chi \in \Xi$ we define $V_{\chi}:=\{v \in V \mid a v=\chi(a) v\}$ for all $a \in \mathcal{H} \otimes \mathbb{C}\left[\mathbb{F}_{q^{n}}^{*}\right]$. Let $\Xi_{V}=\left\{\chi \in \Xi \mid V_{\chi} \neq\{0\}\right\}$.

Theorem 0.3. (1) $\operatorname{dim}\left(V_{\chi}\right)=1$ for all $\chi \in \Xi_{V}$.

(2) $V=\oplus_{\chi \in \Xi_{V}} V_{\chi}$.

Proof. As follows from [1] and [5] we have direct sum decomposition

$$
V=\oplus_{\chi \in \Xi_{V}} V_{\chi}
$$

where the subspaces $V_{\chi} \subset V$ are $\mathcal{H} \times G_{\infty}$-invariant and the representation $\tilde{\rho}_{\chi}$ of $\mathcal{H} \times G_{\infty}$ on $V_{\chi}$ is irreducible. Since $\mathcal{H}$ is commutative this implies the irreduciblity of the restriction $\rho_{\chi}$ of $\tilde{\rho}_{\chi}$ to $G_{\infty}$. By definition we can consider $\rho_{\chi}$ as a representation of the quotient group $G_{\infty} / K_{\infty}^{1}=\mathbb{Z} \ltimes \mathbb{F}_{q^{n}}^{\star}$ where $1 \in \mathbb{Z}$ acts by the Frobenious automorphism on $\mathbb{F}_{q^{n}}^{\star}$. It is easy to see that the restriction of any irreducible representation of the group $\mathbb{Z} \ltimes \mathbb{F}_{q^{n}}^{\star}$ on $\mathbb{F}_{q^{n}}^{\star}$ is the direct sum of distinct one-dimensional representations.

Question 0.4. Is the subalgebra $\alpha(\mathcal{A}) \subset \mathbb{C}_{c}\left(G_{0}\right)$ invariant under the natural action of the group of automorphisms of $F$ ?

Remark 0.5. The paper [4] was influnced by [3] and is concerned with the understanding of the local Langlands conjecture. This short paper is a streamlined version of [4].

Acknowledgments. The project has received funding from ERC under grant agreement 669655 . 


\section{REFERENCES}

[1] Deligne, P.; Kazhdan, D.; Vign'eras, M.-F. Représentations des alge'bres centrales simples $p$-adiques. Representations of reductive groups over a local field, 33-117, Travaux en Cours, Hermann, Paris, 1984"

[2] Hrushovski, E,; Kazhdan D.; Motivis Poisson summation. Moscow Math. J. 9(2009) no. 3 569-623

[3] Katz, N.Local-to-global extensions of representations of fundamental groups. (French summary) Ann. Inst. Fourier (Grenoble) 36 (1986), no. 4, 69106.

[4] Kazhdan, David On a theorem of N. Katz and bases in irreducible representations. From Fourier analysis and number theory to Radon transforms and geometry, 335340, Dev. Math., 28, Springer, New York, 2013

[5] Piatetskii-Shapiro I. Multiplicity one theorems, Proc. Sympos. Pure Math., vol. 33, Part I, Providence, R. I., 1979, pp. 209-212. 